literacy with some differences in function of word versus non-word reading and writing.

Conclusions These findings revealed the importance to analyse both language and short-term memory in preterms at the end of preschool age, with relevant implications for interventions to improve literacy at school age.

\section{DEVELOPMENT AND VALIDATION OF A SCALE TO ASSESS KNOWLEDGE OF OUTCOMES FOLLOWING PRETERM BIRTH}

doi:10.1136/archdischild-2012-302724.1235

'D Henderson, ${ }^{1} \mathrm{C}$ Beer, ${ }^{2} \mathrm{D}$ Wolke, ${ }^{3} \mathrm{~S}$ Johnson. ${ }^{1}$ School of Community Heath Sciences, University of Nottingham, Nottingham; ${ }^{2}$ Warwick Medical School, University of Warwick, Coventry; ${ }^{3}$ Department of Health Sciences, University of Leicester, Leicester, UK

Background and Aims Preterm children are at high-risk for special educational needs. Education professionals' (EPs) knowledge of health conditions is crucial for providing appropriate support, however no studies have investigated their knowledge in this area. To facilitate such research we developed a scale to assess knowledge of outcomes following preterm birth.

Methods Following a comprehensive literature review, 35 forced choice (true/false/don't know) items were developed to assess knowledge of neurodevelopmental and educational outcomes. Item scores were summed to provide a total knowledge score (range $0-35)$. The scale was completed by 120 EPs and 70 experts in the field (neonatologists/paediatricians).

Results EPs' responses revealed floor effects for 2 items which were removed. The remaining 33-item scale had excellent internal reliability (Cronbach's Alpha=0.82). EPs' knowledge scores were normally distributed (Mean 11.3; SD 5.4) and differed significantly by level of training $(\mathrm{F}(3,111)=2.78, \mathrm{p}=0.045)$ indicating construct validity. Experts' knowledge scores were not normally distributed (Median 26.5; IOR 23.0-29.0) and were significantly higher than EPs $(p<0.001)$ indicating discriminative validity. Principal components factor analysis revealed two factors:

1. developmental problems, internalising difficulties and attainment,

2. need for additional support in the classroom.

EPs' scores were significantly lower than experts on both subscales.

Conclusions This short scale has good psychometric properties and provides a useful tool for teaching and assessing clinical and education professionals' knowledge of preterm birth. Study results revealed the need to improve EPs' knowledge of sequelae of prematurity if they are to support increasing numbers of preterm children in the classroom.

\section{SUPPORTING THE SCHOOLING OF VERY PRETERM CHILDREN: EDUCATION PROFESSIONALS' OPINIONS AND INFORMATION NEEDS}

doi:10.1136/archdischild-2012-302724.1236

${ }^{1} \mathrm{D}$ Henderson, ${ }^{1} \mathrm{C}$ Beer, ${ }^{2} \mathrm{D}$ Wolke, ${ }^{3} \mathrm{~S}$ Johnson. ${ }^{1}$ School of Community Heath Sciences, University of Nottingham, Nottingham: ${ }^{2}$ Warwick Medical School, University of Warwick, Coventry; ${ }^{3}$ Department of Health Sciences, University of Leicester, Leicester, UK

Background and Aims Increasing numbers of very preterm (VP; < 32 weeks) children with special educational needs are entering, and challenging, the education system. At present we know little about teachers' information needs or opinions regarding how to support these children.

Methods Preschool and primary education professionals completed a questionnaire to elicit opinions regarding their information needs, disclosure of birth status and delayed school entry for VP children. Respondents rated how strongly they agreed with 10 statements using a 5-point Likert scale; responses ranged from strongly agree to strongly disagree. Additional questions explored training received and demographic information.

Results Of the 120 respondents, $89 \%$ felt they were likely to teach a VP child and that educational management was the role of the class teacher. However, only $6 \%$ reported having received sufficient training about VP children and $>90 \%$ agreed they would like more information about strategies to support VP children's learning. $92 \%$ of respondents agreed that disclosing a child's preterm birth status would be beneficial and none felt it would lead to problems associated with labelling. A small majority of teachers were supportive of parents delaying $(56 \%)$ or deferring $(58 \%)$ their child's school entry. Conclusions This survey revealed that a worrying number of early years' education professionals received no formal training regarding the educational needs of VP children and most felt inadequately equipped to support these children in their classroom. There is a pressing need for clinicians to communicate evidence-based findings to facilitate ongoing management of VP children at school.

\section{DEVELOPMENT AND QUALITY OF LIFE IN NICU GRADUATES AND HEALTHY NEWBORNS WITH PCHI AT 3-5 YEARS OF AGE AFTER NHS}

doi:10.1136/archdischild-2012-302724.1237

${ }^{1}$ EA van de Ven, ${ }^{1} H L M$ van Straaten, ${ }^{2}$ AM Oudesluys-Murphy, ${ }^{2} \mathrm{AMH}$ Korver. ${ }^{1}$ Dept of Neonatology, Isala Clinics, Zwolle; '2Dept of Pediatrics, Leiden University Medical Center, Leiden, The Netherlands

Background NICU graduates have both an increased risk of permanent childhood hearing impairment (PCHI) and delayed psychomotor development. Little follow up is available after introduction of neonatal hearing screening (NHS).

Aim Comparison of language and psychomotor development as well as quality of life in NICU graduates vs. well baby clinic (WBC) newborns with PCHI at the age of 3-5 years.

Method All children (born 2003-2005) with PCHI identified in the Netherlands at speech and hearing centres were included. Development and quality of life (OOL) were measured at age $3-5$ yrs by using the MacArthur Communicative Development Inventory, Child Development Inventory (CDI), and Pediatric QOL Inventory 4.0.

Results Twenty-six NICU graduates and 64 WBC newborns completed all measurements. Language development showed significant difference for total words signed (23.3 vs 10.6 ; $\mathrm{p}=0,009)$. All other scores in NICU graduates on subscales of MacArthur tend to be lower. Though CDI scores were not statistically significantly different, the scores of social (70.1 vs 75.2), self-help (80.0 vs 86.2 ) and gross motor development (72.0 vs 83.4 ) were considered clinically significant. Total QOL score differed not significantly (79.4 vs 85.5$)$, inclusive physical functioning ( 85.7 vs 90.4 ) but the subscales social functioning (75.4 vs 85.3; $\mathrm{p}=0,004)$ and psychosocial health summary score (75.9 vs $82.4 ; \mathrm{p}=0,018)$ did for NICU graduates.

Conclusion After NHS NICU graduates with PCHI pursuit to have an increased risk for delayed language and psychomotor development compared to their 'healthy' peers with PCHI. Also quality of life is negatively affected.

\section{INCIDENCE OF CEREBRAL PALSY IN PRETERM BORN CHILDREN DID NOT CHANGE SINCE 2004}

doi:10.1136/archdischild-2012-302724.1238

FV van Daalen, EF Stremmelaar, AF Bos. Department of Pediatrics, Division of Neonatology, University Medical Center, University of Groningen, Groningen, The Netherlands

Introduction During the last decade survival rates of preterm children continuously improve. It is under debate whether this influences the incidence of cerebral palsy (CP). 
Objective To determine changes in incidence of CP and motor disorders in preterm children since 2004, and to identify associated factors.

Methods We included all infants born $<32$ weeks, admitted to our tertiary NICU, born in 2004-2005 (period-I) and in 2008-2009 (period-II). We excluded children transferred from other tertiary NICUs, with major malformations, and neuromuscular disorders. We compared the incidence of CP and other motor disorders at the age of 2 years. To identify potential risk factors, we used the Nursery Neurobiologic Risk Score (NBRS), including $\mathrm{pH}$, ventilation, infection, convulsions, intraventricular hemorrhage, periventricular leukomalacia, and hypoglycemia.

Results 558 children were included; 269 period-I and 289 period-II. The incidence in CP was not significantly different: $5.6 \%$ vs $6.6 \%$, respectively. The number of children with other motor disorders was significantly higher in period-I than in period-II: 15 vs $5(5.6 \%$ vs $1.7 \%, p<0.05$ ). NBRS-scores were median 2.5 vs 2.0 ( $p=0.12$ ). In period-I, $\mathrm{pH}$ and infection contributed more to a higher NBRS, whereas mild periventricular leukomalacia did in period-II (all $p<0.05)$. Total and subscores of the NBRS were strongly related to CP $(p<0.01)$, apart from hypoglycemia in both periods, and ventilation and suspected infections in period-I.

Conclusions Since 2004, the incidence of CP in preterm children did not change, but rates of other motor disorders decreased, without considerable changes in associated risk factors.

\section{NEURODEVELOPMENTAL OUTCOME ONE YEAR AFTER EARLY VERSUS LATE SELECTIVE SURFACTANT TREATMENT}

doi:10.1136/archdischild-2012-302724.1239

${ }^{12}$ Eras, ${ }^{2} \mathrm{HT}$ Aksoy, ${ }^{2} \mathrm{R}$ Ozdemir, ${ }^{2} \mathrm{~N}$ Uras, ${ }^{3} \mathrm{U}$ Dilmen. ${ }^{1}$ Developmental Behavioral Pediatrics Unit; ${ }^{2}$ Neonatology, Zekai Tahir Burak Maternity and Teaching Hospital; ${ }^{3}$ Neonatology, Zekai Tahir Burak Maternity and Teaching Hospital, Yıldırım Beyazıt University Department of Pediatrics, Ankara, Turkey

Aim To investigate whether neurodevelopmental outcome at age one year might be different after early versus late rescue surfactant treatment in preterm infants.

Methods In 54 preterm infants, having gestational age between 25-30 weeks who were enrolled in a controlled trial of early versus late selective surfactant treatment (45 vs. 70 min respectively), a standardized follow up of medical history, neurodevelopmental outcome using the Bayley Scales of Infant and Toddler Development, Second Edition at 9-12 months corrected age.scales were carried out.

Results Median Mental developmental index (MDI) score was 107 for early group and 111 for late group. Median Psychomotor developmental index (PDI) score was 82 for early group and 93 for late group. Although median MDI and PDI scores were slightly higher in late poractant treatment group and neurodevelopmental impairment was higher in early rescue group than the late rescue group, this was not statistically significant.

Conclusion Our results demostrated that both early and late poractant treatment had similiar effects on the neurodevelopmental outcomes of preterm infants with RDS. In terms of neurodevelopmental outcomes there is no obvious advantage of an immediate surfactant administration in preterm infants according to our results.

\section{MOTHERS' AND HEALTH PROFESSIONALS' PERSPECTIVES OF BARRIERS AND FACILITATORS TO ATTENDANCE AT CANADIAN NEONATAL FOLLOW-UP PROGRAMS}

doi:10.1136/archdischild-2012-302724.1240

${ }^{1} \mathrm{M}$ Ballantyne, ${ }^{2 \mathrm{~K}}$ Benzies, ${ }^{3} \mathrm{~A}$ Lodha, ${ }^{4} \mathrm{P}$ Rosenbaum. ${ }^{1}$ Nursing, Faculty of Health Sciences, McMaster University, Hamilton, ON; ${ }^{2}$ Faculty of Nursing: ${ }^{3}$ Department of
Paediatrics, University of Calgary, Calgary, AB; ${ }^{4}$ Department of Paediatrics, McMaster University, Hamilton, ON, Canada

Background and Aims Neonatal Follow-Up (NFU) programs are increasingly challenged to support attendance, address family needs, and provide essential services. Up to $30 \%$ of families do not attend these programs. The aim of this research was to investigate barriers and facilitators of NFU attendance from the perspectives of health care professionals (HCPs) and mothers.

Methods A qualitative research approach using purposive maximum variation sampling was conducted. HCPs participated in focus groups; mothers in individual interviews. Descriptive analysis was conducted to determine themes. Data from HCPs and mothers were analyzed separately and then compared.

Results Participants were $20 \mathrm{HCPs}$ from 9 NFU programs and 6 mothers from $2 \mathrm{NFU}$ programs located in the most populous regions of central and western Canada. Both HCPs and mothers identified limited family resources and restrictive clinic operations as barriers. HCPs identified fear of bad news as a barrier; mothers viewed bad news as a facilitator; the need to address the issue and move forward. Both HCPs and mothers viewed vulnerability as a barrier; however, the meaning differed. HCPs reported creating vulnerability for the family by monitoring their child's development over time; whereas, mothers reported the need to protect their vulnerable child from risks (i.e., infection, weather). For mothers, the vulnerable child in combination with limited family resources was a key barrier to attendance.

Conclusions A better understanding of these perspectives may facilitate modifications to NFU programs to increase attendance, and ultimately improve outcomes for children at high risk for developmental delays and their families.

\section{ADAPTATION PROBLEMS IN VERY LOW BIRTH WEIGHT CHILDREN AT 10 YEARS OF AGE}

doi:10.1136/archdischild-2012-302724.1241

${ }^{1} \mathrm{~T}$ Fjørtoft, ${ }^{2,3} \mathrm{KH}$ Grunewaldt, 'M Sognnæs, ${ }^{2,4} \mathrm{GCC}$ Løhaugen, ${ }^{2,3,4} \mathrm{~J}$ Skranes, ${ }^{5,6} \mathrm{KAl}$ Evensen. 'Clinical Services, St. Olavs University Hospital; ${ }^{2}$ Laboratory Medicine, Children's and Women's Health, Norwegian University of Science and Technology; ${ }^{3}$ Paediatrics, St. Olav University Hospital, Trondheim; 'Paediatrics, Sørlandet Hospital, Arendal; ${ }^{5}$ Public Health and General Practice, Norwegian University of Science and Technology; ${ }^{6}$ Physiotherapy, Municipality of Trondheim, Trondheim, Norway

Background Adaptive behaviour is the behaviour necessary for an individual to function safely and appropriately in daily life, both at a personal and social level. The Vineland Adaptive Behavior Scales (VABS) has been used to describe an individual's adaptive behaviour as reported by caregivers.

Objective To compare skills of communication, daily living and socialisation as well as the total behaviour score in very low birth weight (VLBW) children with a control group at ten years of age.

Design/methods In this follow-up study, 39 children with birth weight below 1500 grams, including 10 children with cerebral palsy (CP), and 31 term born control children were evaluated by VABS at ten years of age. The informants were parents, mostly mothers. In VABS, adaptive behaviour is expressed as total adaptive behavior composite score based on the three subscales: Communication, daily living skills and socialisation.

Results The mean total adaptive behaviour composite score in the VLBW group was 85.7 (SD 16.8) compared with 105.5 (SD 17.5) in the control group $(p<0.001)$. All three subscales; communication, daily living skills and socialisation were significantly lower in the VLBW group than in the control group. Excluding children with CP, the total adaptive behavior composite score was 90.6 (SD 14.5) in the VLBW, still significantly lower than in the control group $(\mathrm{p}=0.001)$. 